\title{
XXIX. On spontaneous nucleation and on nuclei produced by shaking solutions
}

\section{Barus}

To cite this article: C. Barus (1902) XXIX. On spontaneous nucleation and on nuclei produced by shaking solutions, Philosophical Magazine Series 6, 4:20, 262-269, DOI: 10.1080/14786440209462843

To link to this article: http://dx.doi.org/10.1080/14786440209462843

曲 Published online: 15 Apr 2009.

Submit your article to this journal $\square$

Џ Article views: 3

Q View related articles $\square$ 
depends upon a knowledge of the law of absorption which we do not at present possess, it is interesting to find that a collection of corpuseles describing circles under forces varying inversely as the square of the distance in the molecules of a substance which shows no selective absorption would, like the ideal "black" body, radiate at a rate proportional to the fourth power of the absolute temperature.

XXIX. On Spontaneous Nucleation and on Nuclei produced by Shaking Solutions. By C. Barus*.

\section{Spontaneous Nucleation.}

$\mathbf{I}^{\mathrm{N}}$

N 'Science' (xr. Jan. 1902, p. 178) I communicated some results which seemed to give evidence of the spontaneous production of nuclei from certain organic liquids. Though my own work is rather more concerned with the diffusion of the nuclei with an ulterior view to their velocity, no matter how the nuclei may be localized, it nevertheless seemed interesting to elucidate the subject incidentally. I therefore made a series of experiments in which condensation was produced by the expansion method in case of gasoline, benzine, petroleum, benzol, carbon bisulphide, and water.

Hydrocarbons.-The first three hydrocarbon liquids may be dismissed summarily. The air above them, if carefully freed from nuclei by precipitation, remained free from nuclei indefinitely. The test was made by leaving the receiver without interference for fifteen or more hours, all the cocks being shut off, except the one communicating with the atmosphere through a filter of compressed cotton, half a metre long. A perfect filter is essential throughout. In case of petroleum it is exceedingly difficult to remove the nuclei by precipitation alone; but they vanish in the lapse of time (days), and thereafter the air remains permanently without nucleation.

In case of benzol I was for a Jong time erroneously of the opinion that nuclei arise spontaneously out of this liquid, and consistent results leading to this inference were obtained in great number. Doubt was cast on this supposition by the behaviour of the hydrocarbons just mentioned. The true explanation was subsequently found: on removing nuclei by precipitation with the objeet of obtaining dust-free air, a couche of nuclei is apt to remain brooding immediately over the surface of the benzol, where it escapes detection. It is

$$
\text { * Communicated by the Author. }
$$


on Nuclei produced by Shaking Solutions.

in this couche that the nuclei which subsequently diffuse * into and fill the whole vessel originate. They do not come out of the benzol.

To account for these couches, which occur more or less frequently with all hydrocarbons and other volatile liquids, it is necessary to consider the manner in which the nuclei are introduced into the receiver. This is done expeditiously by partially exhausting the receiver and allowing the inflowing air to pass over phosphorus, or glowing charcoal, or near a sulphur flame. In the case of water vapour the nuclei after entrance remain permanently apart. The nucleated air is always homogeneous and the coronas regular. Semi-coronas never occur. This indefinite suspension of nuclei means that they remain small, diffuse relatively fast, and gravitate very slowly. The phenomenon is very similar to the suspension of particles of clay in water. The speed of subsidence is a minimum.

In case of the hydrocarbons \&c. the occurrences are very different. What goes on while the nuclei are being introduced is not of course visible; but the first exhaustion after nucleation shows a horizontally graded distribution, in which the nuclei are wholly confined to a narrow stratum, usually immediately above the liquid, as already stated. The fog stratum may, however, show itself at the top of the vessel, or even between two hemispheres of clear non-nucleated air. Indeed the air is rarely, if ever, nucleated uniformly.

The distribution, therefore, is one of density ; and from the relatively insignificant number of nuclei, it may be further supposed that to influence the density of the strata, the nuclei have been loaded on influx, almost without supersaturation, even though the fog particles are small enough to remain invisible. In such a case it is hardly probable that the nuclei have remained individualized as in the case of water vapour ; it is more probable that they grow by coalescence or cohesion, until they are large enough to condense hydrocarbon vapour with the minimum of supersaturation or none at all. This again is remarkably like the subsidence of clay in hydrocarbon liquids, in which, from the cohesion of particles, the precipitation is, relatively speaking, instantaneous.

It is not necessary, however, to assume loading. If the nucleus diffuses slowly enough in organic vapours to virtually

* The rate of diffusion (roughly, 015 centim./sec., upward in benzol vapour, for instance) is the feature of these experiments on which I am now at work. Incidentally cne may note that the "granular" particles in water vapour should diffuse much more rapidly than the "flocculent" particles in benzol vapour, the nuclei being otherwise the same. 
stick to the layers in question, and if there is any slight difference in temperature between the air inside of the receiver and the inflowing air, couches will result from convection. Conversely, the entire non-occurrence of semi-coronas \&c. in case of water vapour is an indication of relatively rapid diffusion, and therefore of nuclei of minimum size. The nucleus depends for its size, cæt. par., on the medium in which it is suspended. Nuclei from the same source will diffuse under otherwise like conditions 100 or 200 times more rapidly in water vapour than in benzol vapour.

Under these conditions there would be fewer particles as compared with water vapour for like nucleation, \&c., and normal coronas may appear at once in volatile hydrocarbons, whereas they are only reached after many exhaustions in water vapour. Experiment * bears this out. Moreover, the particles in the former case being larger require less supersaturation, and are more quickly removed, which is also true of the volatile liquids.

Carbon Bisulphide.--The case of carbon bisulphide is peculiar, inasmuch as in addition to the occurrence of couches of the kind just described, this liquid emits nuclei spontaneously. I would not wish to assert this for the fresh and perfectly pure liquid; but from carbon bisulphide which has been subjected to evaporation and contains sulphur, nuclei are continually evolved at a definite rate. Curiously enough, the addition of nuclei from without, whether coming from sulphur, punk, or atmospheric air (without shaking of the liquid) is almost insignificant. A couche a few centims. high is usually seen on first exhaustion, the remainder of the receiver being clear.

After shaking the first corona is annular, coarse, and normal, the aperture of its white disk $s=3^{\circ} \cdot 7$. The next exhaustion brings out semicircles concave upward or semiannuli, the succeeding exhaustion quarter-annuli, \&c., the colour eventually vanishing in a filmy veil. Left to themselves these partial nucleations gradually diffuse upward, and full coronas may be again obtained in the lapse of time. The same result occurs if the air above the liquid has been quite freed of nuclei by repeated precipitation, as the nuclei are now supplied by the liquid itself. These coronas are first partial, then filmy and small but complete. Gradually they become larger and stronger until a limiting aperture and great intensity is reached. In other words, nuclei are emitted by the liquid in the dark, terminating in a state of saturation

* Cf. American Journ. Sci., (4) xiii. pp. 81-94 (1902). 
on Nuclei produced by Shaking Solutions.

of the air above. The following are typical data; $s$ is the aperture *:-

\begin{tabular}{|c|c|c|c|c|c|c|c|}
\hline & & & & $\min$ & & & \\
\hline & $\begin{array}{l}\text { Film, } \\
\text { high }\end{array}$ & & & $\begin{array}{l}\text { Strong, } \\
s=1^{\circ .8 .}\end{array}$ & $\begin{array}{c}\text { Strong, } \\
s=2^{\circ} \cdot 25 \text {. }\end{array}$ & $\begin{array}{c}\text { Strong } \\
s=3^{\circ} \cdot 4 .\end{array}$ & \\
\hline
\end{tabular}

If the evaporation is accelerated, after shaking, as in a warm bright room, the saturated condition may be reached in two hours or less. Usually the coronas of the first hour, though full, are too faint for measurement. On successive exhaustions, the intense coronas vanish by passing through three-quarter, half, quarter coronas, \&c., in turn.

\section{Persistent Liquid Nuclet.}

Hydrocarbons, \&c. - Nuclei obtained by vigoronsly shaking all the volatile liquids examined above, showed no tendency to vanish until removed by gravity. I will cite special instances at random bearing on this remarkable behaviour. Coronas produced by precipitating gasoline vapour on the nuclei produced by shaking the liquid, were observed with undiminished strength 7 minutes and 30 minutes after shaking, and might have been seen after an indefinitely longer time The average apertures were $s=1^{\circ} \cdot 4$, corresponding to the diameter of particle $d=0029$ centim. In petroleum the coronas were observed strongly an hour after shaking; in benzol similarly intense $5 \mathrm{~min} ., 30 \mathrm{~min}$., $44 \mathrm{~min}$., and $60 \mathrm{~min}$. after shaking; they were distinct 3 hours after but absent next day, about 15 hours after. In carbon bisulphide the coronas due to shaken nuclei coalesce with the coronas due to spontaneous nuclei, so that they persist indefinitely.

From the excessively volatile character of these liquids persistence, even beyond a few minutes, would be impossible if the liquids were quite pure. For the small drops would evaporate and the large drops subside. Consequently the droplets must be solutions obtained by concentrating the impurities throughout the continued evaporation of the liquid by the addition of nuclei, \&c. It is difficult to keep these liquids quite pure in an apparatus of which rubber tubing $\dagger$ is an essential part. Apparatus made entirely of glass parts

* Source of light and goniometer are on opposite sides of the receiver, each 2.5 metres distant. The aperture $s$ subtends the central white disk of the normal corona. If $d$ is the diameter of particle, $d=00144 / s$ centim. nearly.

+ Rubber tubing through which carbon bisulphide has been passed will furnish nuclei to precipitate water vapour for days after. Fresh tubing must therefore be provided for each new experiment. 
fused together would be undesirable for practical reasons. To avoid these dilemmas experiments may be made with water, in which the difficulties mentioned do not occur, and a definite decision may be reached.

Water.-If all precautions be taken to insure clean and new apparatus and appurtenances and perfect filtration, the air above the water, if free from nuclei, remains so indefinitely. Spontaneous nucleation does not occur

If a clean vessel free from nuclei is vigorously shaken so as to comminute the water, nuclei are produced in considerable quantity, as is evidenced by the strong coronas seen on exhaustion. But these coronas are short-lived, as shown by the following table :-

Time elapsed after shaking. $7 \mathrm{~min}$.

$6 \quad$,
$5 \quad$,
3
$2 \quad "$
1,
$\frac{1}{2} "$
None

Corona.

All but absent.

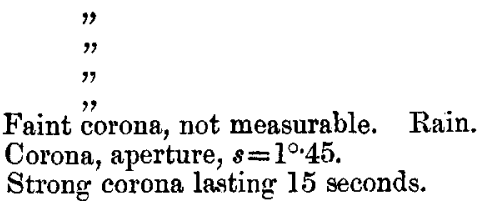

These nuclei are obviously water globules, which speedily evaporate or subside, vanishing in a few minutes.

Let a small quantity of sodic carbonate be added to the water ; the result is a phenomenal increase in the persistence of the nuclei produced by shaking, as for instance :

Time elapsed after shaking.

1 hour.

2,

3 ,

15,

15,

15 " (violent shaking.)
Corona.

$$
\begin{aligned}
& \text { Strong. } \quad s=1^{0.4} \\
& \qquad ", \quad s=1^{\circ} \cdot 6 \\
& \text { ", } \quad s=0^{\circ} .9 \\
& \text { Just visible. } \\
& \text { Absent. } \\
& \text { Distinct, clear, small. }
\end{aligned}
$$

The size and persistence depends on the violence of shaking, the apertures varying from $s=1^{\circ} \cdot 4$ to $s=2^{\circ} \cdot 0$ correspondingly. In the lapse of time the corona contracts, but may, in favorable cases, be seen much after 15 hours.

The body in solution in this case is solid, and one is liable to conclude that the nucleus in question is the solid residue left after evaporation. This, as will presently be shown, is not necessarily true; the nucleus is liquid, being a more or less concentrated solution both here and in the above experiments with hydrocarbons, \&c.

To decide the question as to the cause of the indefinite persistence of the nuclei produced by shaking solutions (apart 
from gravitation), it is necessary to test a chemically dissolved gas like hydrochloric acid or ammonia. Pure water was first taken and the evanescence of the nuclei (say within 4 minutes) confirmed. Pure hydrochloric acid was then added, and the persistence of the nuclei established, as shown by the following example :-

Time elapsed after shaking dilute $\mathrm{HCl}$.

$$
\begin{aligned}
& 10 \% " \\
& 10 \text { min. } \\
& 30 \text { " } \\
& 15 \text { hours. }
\end{aligned}
$$

Corona.

Strong. $s=1^{\circ} \cdot 65$

Strong.

Distinct. Weak.

Tested with pure water again, the fleeting coronas persisted somewhat longer (vanished in 10 ininutes) than before, showing that traces of $\mathrm{HCl}$ still lingered in the clean apparatus. On again adding $\mathrm{HCl}$ the above results were confirmed.

Dilute ammonia was next tested in the same way. Great care had to be taken after the vessel was thoroughly cleaned to remove lingering traces of $\mathrm{HCl}$. When this was done air free from nuclei remained so above dilute ammonia indefinitely. No nuclei arise spontaneously from the liquid.

On shaking the ammonia nucleus behaved like the nucleus of dilute hydrochloric acid, or of sodic carbonate solution, showing indefinite persistence apart from removal by gravity.

With these experiments the indefinite persistence of nuclei produced by shaking solutions, whatever be the original state of aggregation of the solute, may be considered established. It is finally necessary to inquire into the reasons.

Cause of Persistence.-Inasmuch as the nuclei are equally persistent, no matter whether the solute is gaseous or whether it is liquid or solid, it is not permissible to accentuate the possible occurrence of solid residues as the cause of persistence. At first sight one would be liable to conclude that the persistent nuclei are loose molecular aggregates; but their size, which probably embraces many thousands of molecules, is unfavourable to such an assumption. They cannot owe their condensational activity to ionization, for the effect is equally marked with the most pronounced hydrocarbon and other insulators. Frictional electricity seems equally negligible; it would not account for the difference of behaviour of pure water and solutions ; for the electric field within the receiver is a closed field.

The endeavour must therefore be made to explain the phenomenon in terms of surface-tension. If $p_{r}$ and $p_{\infty}$ be the vapour-pressures corresponding to radii $r$ and $\infty$, T the 
surface-tension, $\rho$ and $\sigma$ the densities of liquid (solution) and vapour, Kelvin's equation asserts

$$
p_{r}-p_{\infty}=2 \mathrm{~T} \sigma / \rho r \text {, nearly, if } \rho \text { replaces }(\rho-\sigma) .
$$

The present liquid, however, is a solution and its vapour-pressure, $p_{\infty}$, lies below the normal value for the pure liquid.

Furthermore, as the large drops grow at the expense of the smaller, the vapour-pressure for capillary reasons alone would decrease in the former case and increase in the latter case. At the same time, however, the larger drops becoming more dilute, increase their vapour-pressure, while the smaller drops becoming more concentrated decrease it correspondingly. The second group of influences therefore tend to counteract the effect of capillarity. It is quite conceivable that a state of equilibrium may eventually be reached in which the drops, large and small, will all have the same vapourpressure (namely, that of the free surface of the liquid), that further evaporation will thereafter cease and the droplets persist, however small.

In fact, after the lapse of time the preceding equation will be replaced for a given drop by

$$
p_{r^{\prime}}^{\prime}-p_{\infty}^{\prime}=2 \mathrm{~T}^{\prime} \sigma / \rho^{\prime} r^{\prime}
$$

since the pressure, tension, size, and density of the droplet of solution have all changed by evaporation.

If the more concentrated droplet of radius $r^{\prime}$ now persists, $p_{r^{\prime}}^{\prime}=p_{\infty}$, the vapour-pressure at the flat surface of the original solution. Hence

$$
p_{\infty}-p_{\infty}^{\prime}=2 \sigma \mathrm{T}^{\prime} / \rho^{\prime} r^{\prime}
$$

For the case in which a solid is dissolved its mass remains fixed within each suspended drop while the water alone evaporates. When a gas like $\mathrm{HCl}$ or $\mathrm{H}_{3} \mathrm{~N}$ is dissolved the same will also probably be true, for the receiver is filled to the full partial pressure with the gas in question. Hence $\rho^{\prime}$ may be expressed in terms of $\rho$, or if $c=r^{3} / r^{\prime 3}$,

$$
\rho^{\prime}=1+(\rho-1) \cdot c,
$$

whence finally, if $\delta p=p_{\infty}-p_{\infty}^{\prime}$,

$$
r^{\prime}=2 \sigma \mathrm{T}^{\prime} / \overline{(1+(\rho-1) \cdot c) \cdot \delta p} .
$$

For dilute solutions, $\rho=1$, and the last equation is simply $r^{\prime}=2 \sigma \mathrm{T}^{\prime} / \delta p$.

Hence the nuclei produced by shaking dilute aqueous 
solutions may be considered as subject to this condition, in which all sizes of particles are represented. If $r^{\prime}=\infty$, $p_{\infty}^{\prime}=p_{\infty}$, the vapour-pressure at the flat surface.

Neglecting the factor for electrolytes, if $\delta p$ is expressed in terms of Raoult's law, $n / N$ and $n / \mathbf{N}^{\prime}$ being the original and final ratios respectively, of the numbers of molecules in solute and solvent, and $p$ the vapour-pressure at the flat surface of the reservoir of solution,

$$
\delta p=p\left(n / \mathrm{N}^{\prime}-n / \mathrm{N}\right) /\left(1+n / \mathrm{N}^{\prime}\right) .
$$

Furthermore, if the law discovered empirically by Quincke* $(\mathrm{T}=83+187 n / \mathrm{N}$ dynes) be adduced,

$$
\mathrm{T}^{\prime}=\mathrm{T}+187\left(n / \mathrm{N}^{\prime}-n / \mathrm{N}\right)
$$

where $\mathrm{T}$ is the initial and $\mathrm{T}^{\prime}$ the final surface-tension in dynes per centim.

Thus, finally, if $\left(n / \mathrm{N}^{\prime}-n / \mathrm{N}\right)=k$, for brevity,

$$
n^{\prime}=2 \sigma(\mathrm{T}+187 k)\left(1+n / \mathrm{N}^{\prime}\right) / \overline{p \cdot(1+(\rho-\overline{1}) \bar{c}) k} .
$$

Supposing the electrolytic factor supplied, this equation expresses the relation of the radius of the droplets in terms of the original and final ratios of the numbers of molecules of solute and solvent in solution. It implies that if the nuclei produced by shaking are originally of different sizes this difference will be accentuated. There is thus a curious discrepancy between equation and observation; coronas produced from nuclei due to shaking are always annular on first exhaustion, whatever be the vapour or however long the lapse of time. They eventually vanish annularly, in case of the most volatile hydrocarbon liquid. This can only occur if the shaken nuclei are of about the same size originally, which, in fact, is approximately seen on trial. A reason for it, however, seems difficult to discern. The fact that mere agitation $\dagger$ of the liquid should leave nuclei in its wake so nearly of a size as to produce coronas at all is the essential question.

Brown University, Providence, R.I., U.S.A

* Winkelmanu's Handbuch, vol. i. p. 466 (Breslau, 1891). An excellent account of the capillary coefficients of solutions will here be found, due to Prof. F. Braun.

† Lenard's experiments (Wied. Ann. xlvi. p. 584, 1892) on the electricity of waterfalls might be recalled. 